\title{
Electro-optic correlator for large-format microwave interferometry: Up-conversion and correlation stages performance analysis
}

D. Ortiz, Francisco J. Casas, R. Ruiz-Lombera, and J. Mirapeix

Citation: Review of Scientific Instruments 88, 044702 (2017); doi: 10.1063/1.4979811

View online: http://dx.doi.org/10.1063/1.4979811

View Table of Contents: http://aip.scitation.org/toc/rsi/88/4

Published by the American Institute of Physics

\section{Articles you may be interested in}

Note: Energy calibration of a femtosecond photoelectron imaging detector with correction for the ponderomotive shift of atomic ionization energies

Review of Scientific Instruments 88, 046101046101 (2017); 10.1063/1.4979799

A simple digital control system with field-programmable gate array for stabilization of $\mathrm{CO} 2$ laser output Review of Scientific Instruments 88, 043105043105 (2017); 10.1063/1.4979813

Cold wire constant voltage anemometry to measure temperature fluctuations and its application in a thermoacoustic system

Review of Scientific Instruments 88, 044904044904 (2017); 10.1063/1.4979823

Absolute optical responsivity down to the photon counting level with a photomultiplier tube

Review of Scientific Instruments 88, 043104043104 (2017); 10.1063/1.4979812

A direct differential method for measuring thermal conductivity of thin films

Review of Scientific Instruments 88, 044901044901 (2017); 10.1063/1.4979163

Determination of the resistivity anisotropy of orthorhombic materials via transverse resistivity measurements

Review of Scientific Instruments 88, 043901043901 (2017); 10.1063/1.4978908

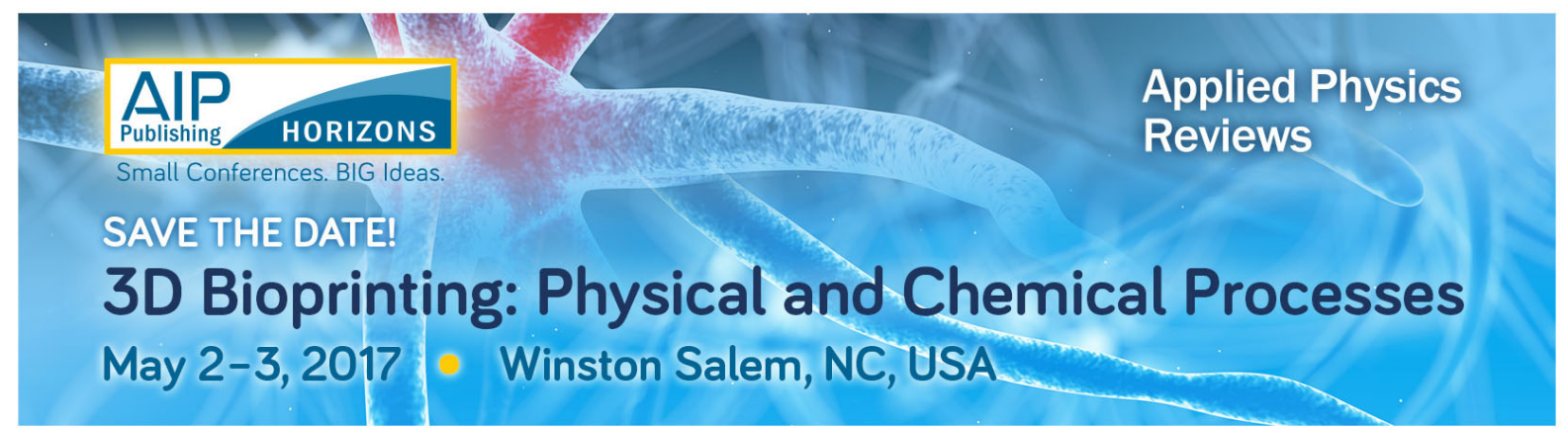




\title{
Electro-optic correlator for large-format microwave interferometry: Up-conversion and correlation stages performance analysis
}

\author{
D. Ortiz, ${ }^{1, a)}$ Francisco J. Casas, ${ }^{1}$ R. Ruiz-Lombera, ${ }^{2}$ and J. Mirapeix ${ }^{2}$ \\ ${ }^{1}$ Instituto de Física de Cantabria (IFCA), Avda. Los Castros s/n, Santander 39005, Spain \\ ${ }^{2}$ Grupo de Ingeniería fotónica (GIF), Plaza de la Ciencia s/n, Santander 39005, Spain
}

(Received 1 February 2017; accepted 24 March 2017; published online 10 April 2017)

\begin{abstract}
In this paper, a microwave interferometer prototype with a near-infra-red optical correlator is proposed as a solution to get a large-format interferometer with hundreds of receivers for radio astronomy applications. A $10 \mathrm{Gbits} / \mathrm{s}$ Lithium Niobate modulator has been tested as part of an electro-optic correlator up-conversion stage that will be integrated in the interferometer prototype. Its internal circuitry consists of a single-drive modulator biased by a SubMiniature version A (SMA) connector allowing to up-convert microwave signals with bandwidths up to $12.5 \mathrm{GHz}$ to the near infrared band. In order to characterize it, a $12 \mathrm{GHz}$ tone and a bias voltage were applied to the SMA input using a polarization tee. Two different experimental techniques to stabilize the modulator operation point in its minimum optical carrier output power are described. The best achieved results showed a rather stable spectrum in amplitude and wavelength at the output of the modulator with an optical carrier level $23 \mathrm{~dB}$ lower than the signal of interest. On the other hand, preliminary measurements were made to analyze the correlation stage, using $4 \mathrm{f}$ and $6 \mathrm{f}$ optical configurations to characterize both the antenna/fiber array configuration and the corresponding point spread function. Published by AIP Publishing. [http://dx.doi.org/10.1063/1.4979811]
\end{abstract}

\section{INTRODUCTION}

During the last decades, ultra-sensitive radio astronomy instruments have been used to characterize the Cosmic Microwave Background (CMB). The CMB is the thermal radiation left over from the time of recombination after Big Bang that was hypothesized by Gamow, Alpher, and Herman in the late $1940 \mathrm{~s}^{1}$ and later accidentally discovered by American radio astronomers, Penzias and Wilson in $1964 .^{2}$ Since then, some ground-based experiments ${ }^{3-7}$ and space missions ${ }^{8-11}$ have been dedicated to the analysis of temperature and polarization anisotropies of the CMB at different frequency ranges. There is also some future experiments ${ }^{12-14}$ trying to improve the sensitivity reached by actual experiments with the aim of measuring the B-mode polarization pattern predicted by inflationary models of the early Universe.

Most of the present active experiments operate as direct image telescopes whose number of receivers is limited by the space available in the focal plane. Therefore, alternative ways to achieve better sensitivities must be considered, especially in the lower bands of the microwave range $(10-50 \mathrm{GHz})$. In particular, the construction of an interferometer overcomes the space limitation of the direct image telescopes by potentially correlating a much larger number (hundreds or even thousands) of elements.

However, the number of elements in CMB interferometers has been usually reduced due to the limitations of traditional analogical correlators in terms of phase controlling and routing a high number of wide-band microwave signals. Other options

\footnotetext{
a) Author to whom correspondence should be addressed. Electronic mail: ortizgd@ifca.unican.es
}

like digital correlators were also discarded because they would need an unaffordable number of digitizing cards, as Fieldeffect Programmable Gate Arrays (FPGAs), to cover the high number of wideband signals required in $\mathrm{CMB}$ experiments.

A novel alternative already implemented in embedded instruments designed for security and defense ${ }^{15}$ is the use of electro-optical correlators that allow a drastic reduction in the complexity of correlating a big amount of wideband microwave signals. The goal of the reported work is to explore the viability of an electro-optic correlator for a future largeformat interferometer intended to characterize the lower frequency bands of CMB. In particular, a correlator prototype to cover a bandwidth from 10 to $12 \mathrm{GHz}$ is being developed. This reduced bandwidth is due to the use of low-cost modulators, required to overcome budget limitations. This correlator is part of an interferometer prototype with four receivers expected to be installed at Teide Observatory (TO) in Tenerife (Spain). This kind of interferometers provides a synthetized image of the polar parameters in the sky region determined by the instrument Field of View (FoV), which is mainly determined by the beam of the horn antennas.

This work describes and analyzes the stability and the carrier suppression level achieved in the modulators of the upconversion stage by implementing a closed-loop feedback ${ }^{16}$ using a photodiode at the output of the modulator and both, a commercial bias controller or a simple lock-in amplifier circuit, to generate the modulators bias signal. On the other hand, in order to have a wider vision about the interferometer operation, some preliminary tests were made in order to characterize the near-infra-red (NIR) correlation process held by optical components.

The document is divided into seven sections. The first one is an introduction followed by a description of the prototype 
in Section II. Section III focuses on the modulator analysis, and Section IV describes the experimental characterization. Section $\mathrm{V}$ provides the results achieved using two different techniques to stabilize the DC bias point of the modulator. Section VI describes the optical setup implemented for preliminary measurements related to the NIR correlation stage. Finally, Section VII draws general conclusions of this work.

\section{INTERFEROMETER PROTOTYPE DESCRIPTION}

The prototype reported in this work will be composed of four microwave receivers covering the $10-20 \mathrm{GHz}$ frequency band and the proposed NIR correlator. Because of the existence of a prohibited frequency band from 14 to $16 \mathrm{GHz}$ at $\mathrm{TO}$ due to interferences, the incoming signal is divided into two sub bands, 10-14 GHz and 16-20 GHz, respectively. Following the scheme of the QUIJOTE experiment instrumentation, ${ }^{17}$ the four output signals from each microwave receiver are proportional to combinations of Stokes parameters. Afterwards, in the correlator up-conversion stage, these microwave signals will modulate the signal of a SFLS1550S laser diode. The selected laser has a linewidth lower than $100 \mathrm{kHz}$, a maximum optical power of $40 \mathrm{~mW}$, and a center wavelength of $1550 \mathrm{~nm}$. Its performance was evaluated using a TEC controller in order to find a stable, temperature and current, operation point free from multi-mode behavior. With this characterization, we fixed $27^{\circ} \mathrm{C}$ and $320 \mathrm{~mA}$ as the working point. In this situation, the optical power at the output of the laser is $24 \mathrm{~mW}$ (Fig. 1).
Once selected a stable operating point of the laser diode, its output signal is divided by optical couplers and introduced at modulator inputs. Later, microwave signals will be up-converted and correlated by optical components to measure the polarization of CMB radiation from the sky, getting the Q, U, and I Stokes parameters of the incoming signal simultaneously. The analysis of the modulator performance and the correlation stage are described Sections III-V. A simplified schematic of the final prototype is shown in Fig. 2.

\section{MODULATOR ANALYSIS}

The modulator selected to implement the up-conversion stage was X-2623Y from Lucent Technologies with an operating wavelength of $1550 \mathrm{~nm}$ and a typical microwave bandwidth of $10 \mathrm{GHz}$.

\section{A. Bandwidth}

Since the modulator bandwidth specifications, it was used to implement the up-conversion stage for the lower-frequency band of the prototype. Its internal circuitry consists of a singledrive modulator biased by a SubMiniature version A (SMA) connector allowing to up convert the microwave input signals from the back-end of the receivers to the NIR band. Fig. 3 shows the modulator output spectrum when a $10 \mathrm{GHz}$ tone is applied and the attenuation of the up-converted microwave signal while frequency increases from 10 to $14 \mathrm{GHz}$ when

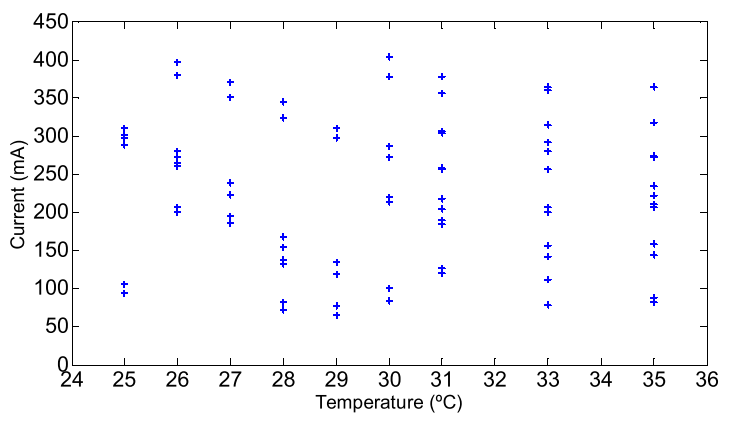

(a)

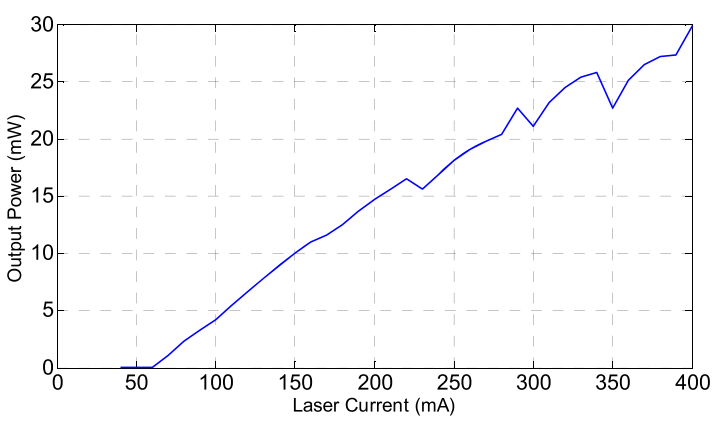

(b)

FIG. 1. Laser diode characterization. (a) Multi-mode behavior ranges. (b) Output power vs. laser current.
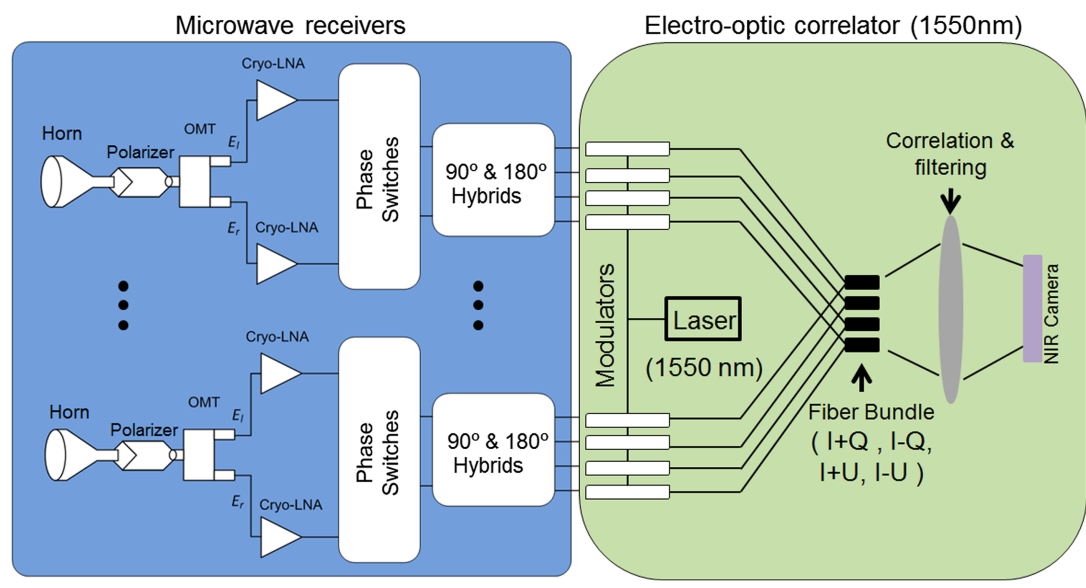

FIG. 2. Simplified sketch of the 10-20 GHz prototype for the impact analysis of the up-conversion and correlation stage behavior. 


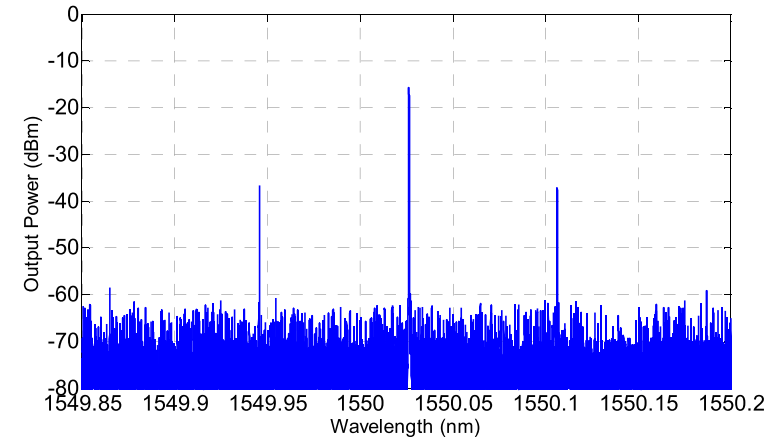

(a)

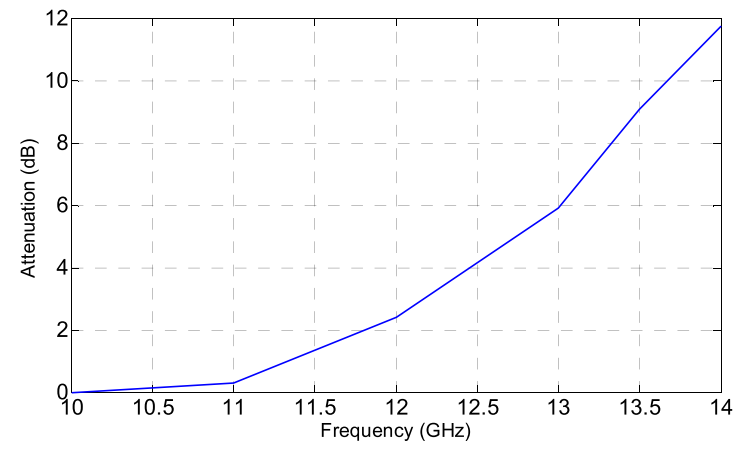

(b)

FIG. 3. Modulator bandwidth performance. (a) Output spectrum (10 GHz). (b) Sideband attenuation (referred to the $10 \mathrm{GHz}$ Spectrum).

the modulator is not DC biased. As the measured attenuation increases a lot with frequency, it was decided to characterize the modulator using a $12 \mathrm{GHz}$ tone, in coincidence with the center of the lower frequency band.

\section{B. DC bias}

Depending on the application, the modulator can be DC biased or not. For the particular application of this work, it is very important to suppress the optical carrier at the output of each modulator as much as possible, while operating in a stable DC bias point. This means that the carrier and up-converted signal levels should maintain the same values during the overall operation time of the instrument, taking into account that this kind of instrumentation operates during large periods of time to get the required measurement sensitivity.

\section{Stability vs time}

The stability of the modulators performance will define the correlator quality, since the instrument will be calibrated at the starting of its operation and the achieved performance should be maintained during the observation period. If not, high errors in phase or amplitude will appear producing a reduction in the quality of the scientific results. ${ }^{18}$ Consequently, keeping a stable output spectrum vs. time will reduce the complexity of analyzing the data at the output of the correlator.

\section{EXPERIMENTAL CHARACTERIZATION}

The up-conversion stage for one receiver output signal was implemented to characterize the stability and the carrier suppression level that could be achieved using one of

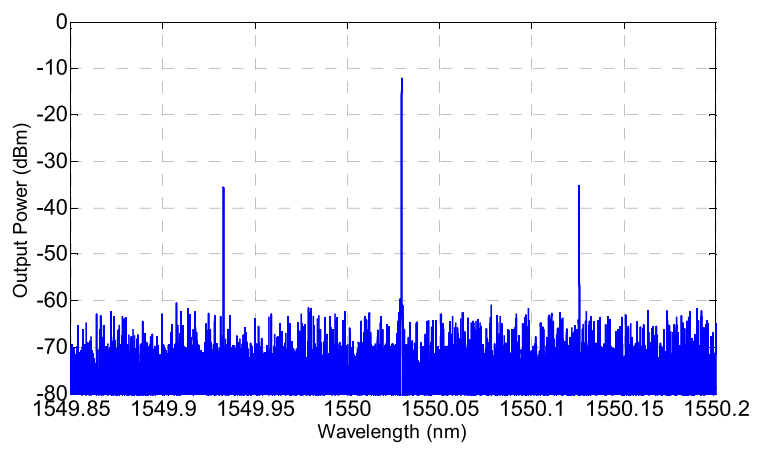

(a)

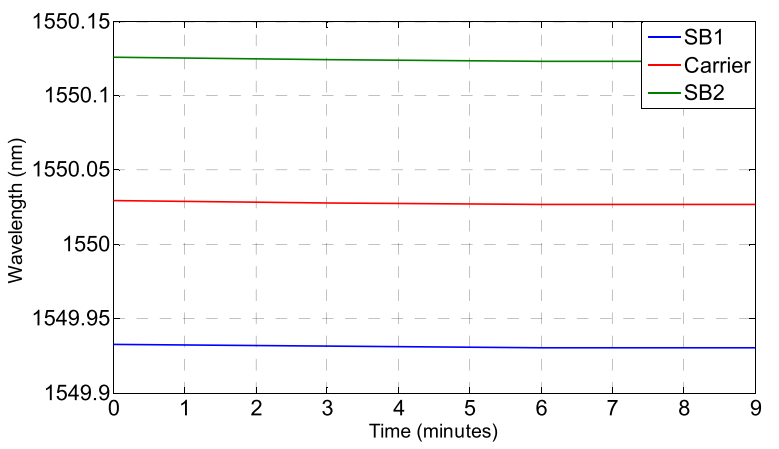

(b)

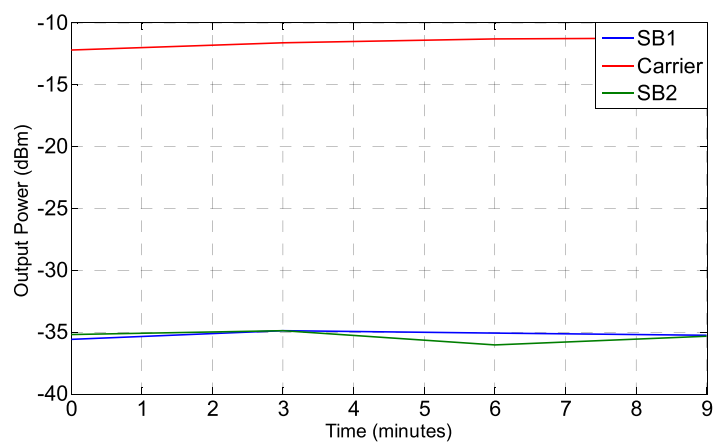

(c)

FIG. 4. Modulator output spectrum. (a) $12 \mathrm{GHz}$ modulation. (b) Wavelength vs. time. (c) Power vs. time. 


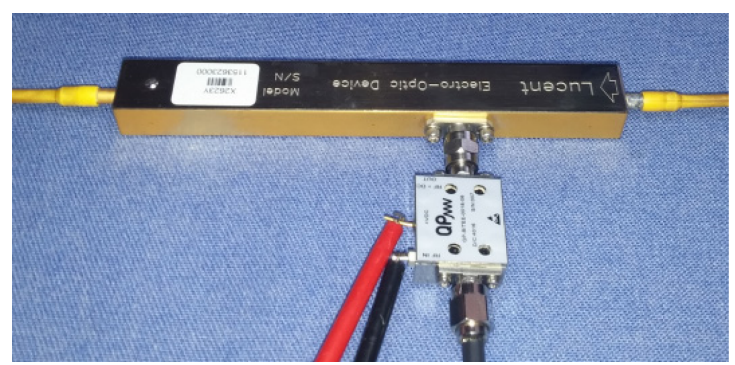

(a)

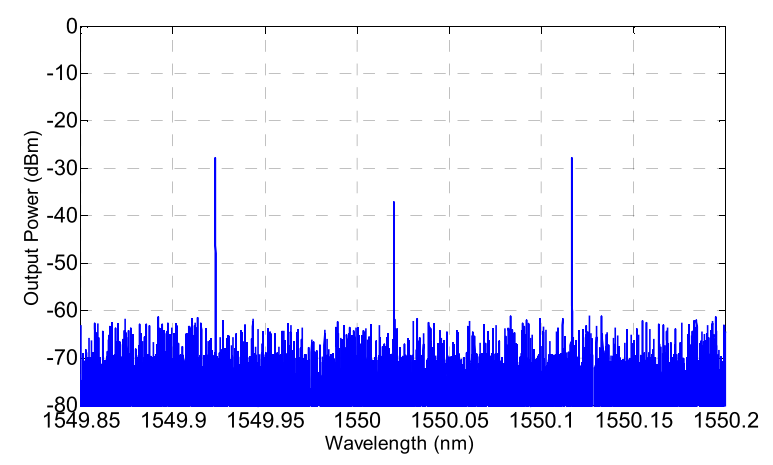

(c)

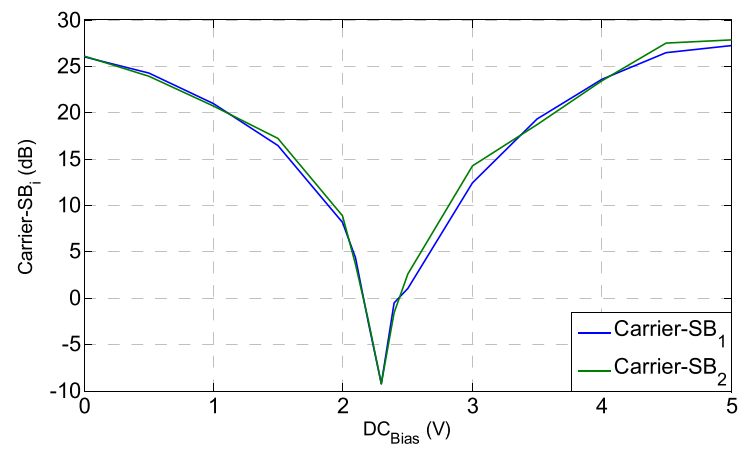

(b)

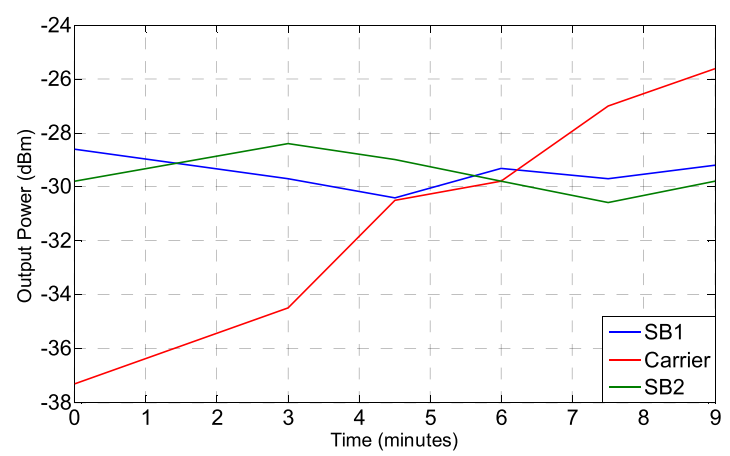

(d)

FIG. 5. Impact of DC bias. (a) Photograph of the modulator and the bias tee. (b) Carrier to sidebands ratio vs. bias voltage. (c) Output spectrum (DC: 2.3 V). (d) Output spectrum vs. time.

the sixteen modulators that will implement the complete upconversion stage. In order to symbolize this scenario, the signal from the laser was introduced into an optical attenuator which helps to modify the incoming optical power. After the attenuation stage, a $1 \times 4$ optical coupler was used to represent the distribution of the laser signal to all the modulators that will compose the final prototype. As the optical components are not fused yet, commercial transitions were used to interconnect them, but their losses are negligible for these tests results.

First measurements were made without DC biasing the modulator. Since the microwave receivers are still under designing phase, a $12 \mathrm{GHz}$ and $10 \mathrm{dBm}$ tone was reproduced by an Agilent Technologies E8257D signal generator. Biasing the laser diode in the selected bias point, the optical power at the input of the modulator was $8 \mathrm{dBm}$.

With this configuration and using a Brillouin Optical Spectrum Analyzer (BOSA) with a high spectral resolution of $0.082 \mathrm{pm}$, the output spectrum of the modulator is shown in Fig. 4. The separation between the carrier and each sideband is $0.096 \mathrm{~nm}$, corresponding to the $12 \mathrm{GHz}$ signal at the input of the modulator.

Once obtained the NIR spectrum, it was measured several times in a period of $9 \mathrm{~min}$ in order to analyse its wavelength and power stability. Figs. 4(b) and 4(c) show the results from this characterization. In the case of wavelength stability, the modulator presented variations lower than $1 \times 10^{-3} \mathrm{~nm}(0.125$ $\mathrm{GHz}$ ). Meanwhile, the power spectrum variations were lower than $\pm 1 \mathrm{~dB}$.
Afterwards, a DC bias was applied at the SMA connector using a polarization tee (Fig. 5(a)). This new setup is intended to reduce the optical carrier from the infrared spectrum. Maintaining the $12 \mathrm{GHz}$ modulation, a sweep varying DC bias in a range from 0 to $5 \mathrm{~V}$ was performed in order to study the carrier suppression levels that could be achieved with respect to the up-converted images of the microwave signals. Fig. 5(b) shows the power difference between the carrier and both sidebands vs. DC bias, and Fig. 5(c) represents the output spectrum obtained in the DC bias optimal point $(2.3 \mathrm{~V})$ where the carrier is near $10 \mathrm{~dB}$ lower than the sideband images. This result was very promising, but the modulator operating point was not stable in time, mainly due to the influence of internal temperature variations induced by the bias voltage itself (Fig. 5(d)). As a consequence, some kind of stabilization method was required to achieve a good level of carrier rejection during large periods of time.

\section{STABILIZATION TECHNIQUES}

After analyzing the behavior of the modulator setting the DC bias manually, the up-conversion stage was modified adding an inline optical power monitor photodiode at the output of the modulator in order to implement a closed-loop feedback for the bias system. This element generates a DC output voltage proportional to the input NIR power. This output signal is then used as a feedback to stabilize the modulator operating point and minimize the optical carrier by two different techniques. 


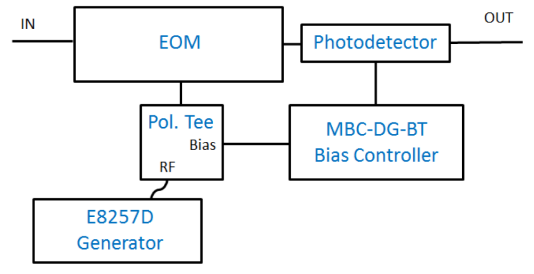

(a)

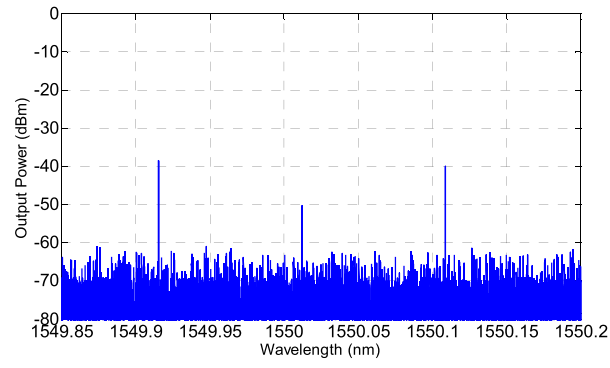

(b)

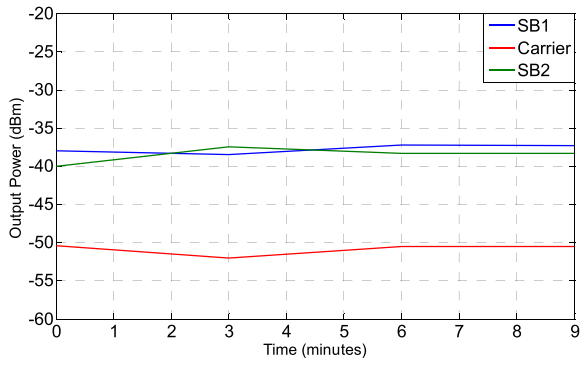

(c)

FIG. 6. MBC-DG-BT bias controller. (a) Feedback close-loop. (b) Output spectrum. (c) Power stability vs. time.

\section{A. Commercial bias controller}

The signal from the photodetector was introduced in a commercial bias controller (model MBC-DG-BT from Photline). The output signal from the photodetector was introduced in the controller to get a stabilized DC bias that was injected to the modulator to close the feed-back loop (Fig. 6(a)). With this new setup, the optimal DC bias point was automatically adjusted to $2.23 \mathrm{~V}$ giving the output spectrum shown in Fig. 6(b). This measurement shows the advantages of using a feedback closed-loop, getting a stable spectrum in wavelength and amplitude, and suppressing $36 \mathrm{~dB}$ the optical carried with respect to the case in which the DC bias was not used. With this configuration, the carrier level was $13 \mathrm{~dB}$ lower than the microwave signal. To this point, it would be necessary to use a filtering technique in the correlation stage in order to reject as much as possible the remaining optical carrier and one of the two sidebands from the modulated NIR signals.

\section{B. Lock-in amplifier}

Since each bias-controller is only able to stabilize a single modulator, the previous solution is not viable for the particular application of this work, taking into account the number of signals to control (4 signals per receiver) and the cost of the bias controllers. As an alternative to the use of commercial DC bias controllers, another solution using the ADA2200 evaluation card from analog devices (Fig. 7) is proposed. This

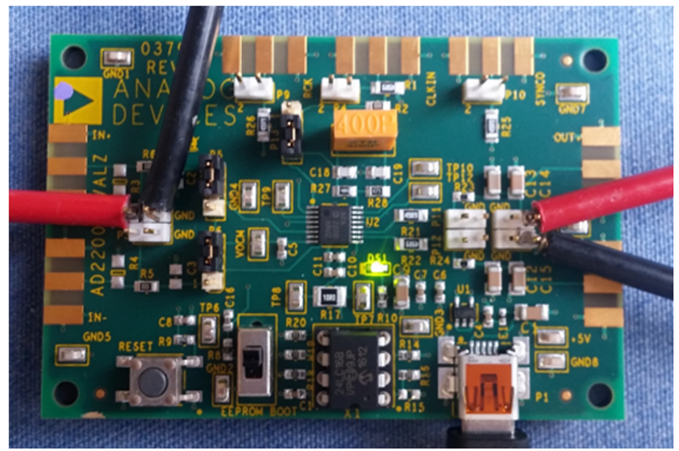

(a)

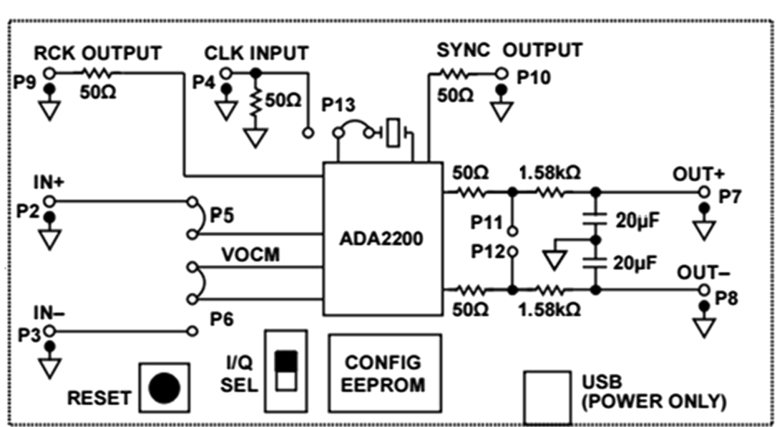

(b)

FIG. 7. ADA2200 evaluation card. (a) Detailed photograph. (b) Configuration by default.

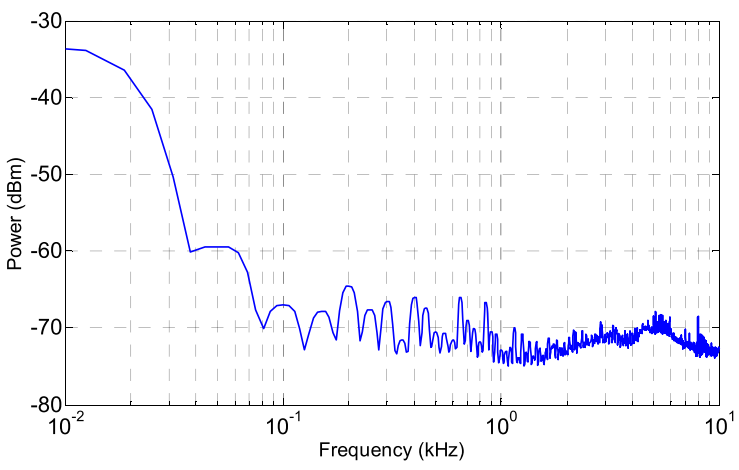

(a)

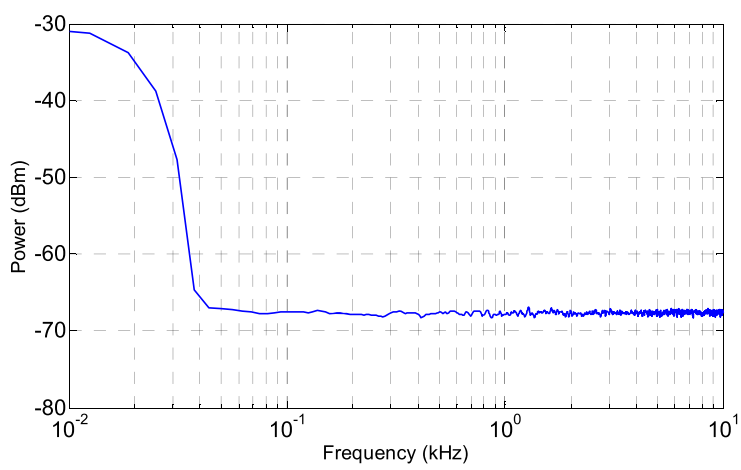

(b)

FIG. 8. Photodetector output signal. (a) Before amplifying. (b) After amplifying. 


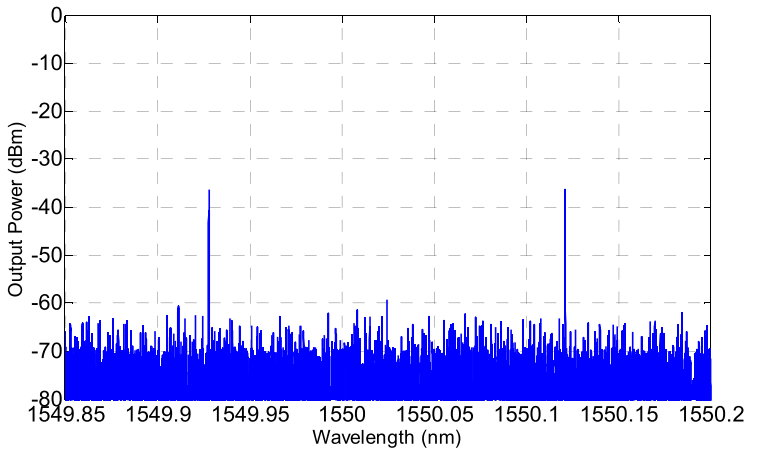

(a)

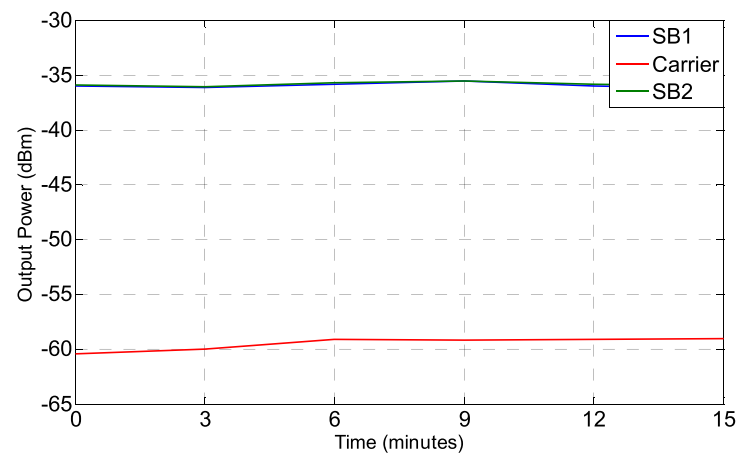

(b)

FIG. 9. Lock-in amplifier feedback. (a) Output spectrum. (b) Power stability vs. time.

card is based on a synchronous demodulator with a configurable analogue filter designed to make low power magnitude and phase measurements with a very high precision. Besides, this card acts as a low-cost lock-in amplifier and allows the implementation of a feedback closed-loop to lock the bias of the modulator in a stable optimal point.

In order to implement the feedback stage using the lock-in, three signals are needed: the output from the photodetector, the lock-in amplifier reference signal, and a DC bias. The output signal from the photodetector is amplified using the evaluation card as a lock-in amplifier, by setting-up the card in its default configuration. Fig. 8 shows the photodetector output signal before and after being amplified by the ADA2200 evaluation card.

The previous signal is then added to the reference obtained from the evaluation card Reference Clock (RCK) output and to the initial bias signal. The reference is a $3.2 \mathrm{~V}_{\mathrm{pp}}, 6.25 \mathrm{kHz}$ squared signal that produces a small modulation on top of the optical pulses to ensure an optimal detection.

Applying the overall signal to bias the modulator, the output signal alternated between two states at a rate fixed by the reference signal. A stable operating point was impossible to be achieved by varying the amplitude or the frequency of the reference signal. For this reason, it was decided to implement a passive feedback without using the reference signal. Using only a DC bias added to the output of the lock-in, it was achieved by a very stable operating point getting $46 \mathrm{~dB}$ carrier suppression at the output of the modulator. With this solution, the optical carrier was $23 \mathrm{~dB}$ lower than the sidebands. Fig. 9 shows the results obtained in a period of $15 \mathrm{~min}$ time.

Although this last result is very promising, it would be necessary again for the introduction of a filtering technique in the correlation stage in order to remove the undesirable components from modulator outputs.

\section{CORRELATION STAGE}

Up to now, since the lack of a Volume Bragg Grating (VBG) optical filter to remove the undesired spectrum components from the up-converted signals, correlation stage preliminary measurements were made using directly the laser diode as a source signal. This signal was divided through optical couplers in order to be distributed and introduced into a bundle. The signals from the output of the bundle were correlated by a couple of $100 \mathrm{~mm}$ focus lenses and finally detected by a Xenics Xeva NIR camera whose thermo-electrically cooled InGaAs detectors are able to detect very weak optical signals, with powers lower than $-60 \mathrm{dBm}$. The implemented setup to characterize the correlation stage is shown in Fig. 10.

In order to group the signals from the $1 \times \mathrm{N}$ coupler, a hexagonally packed bundle with a fixed pitch array of $250 \mu \mathrm{m}$ was designed (Fig. 11). It was decided to fabricate a 46 element bundle in order to have flexibility to optimize the distribution of different 2D antenna array scenarios for the prototype. Although the correlator prototype will have to combine 16 signals (4 groups of 4), there were 20 signals available from the coupling stage to connect to the bundle for preliminary tests.

The bundle should directly map the position of the receiver antennas in a scaled fashion (homothetic mapping). ${ }^{19}$ This means that the optical fibers must be precisely positioned to match the antenna array, thereby necessitating the ability to precisely inject the modulated signals in an array of fibers

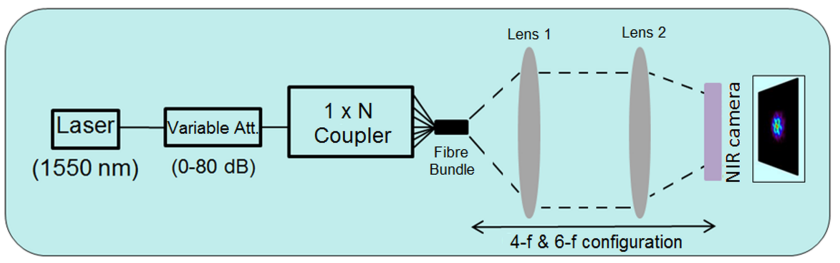

FIG. 10. Sketch of the correlation stage setup.

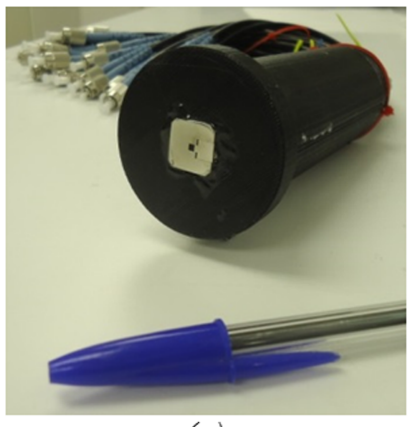

(a)

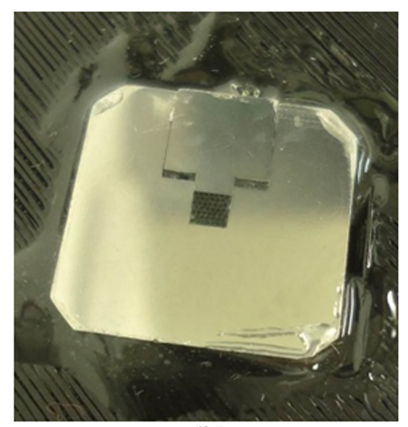

(b)
FIG. 11. Fiber bundle. (a) 46 element fiber bundle. (b) Bundle output (detailed photograph). 


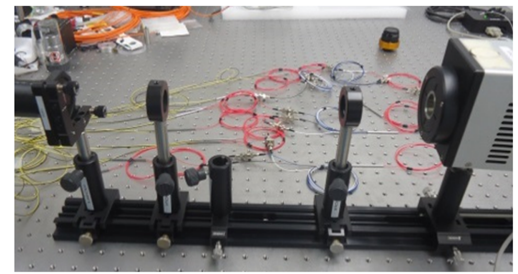

(a)
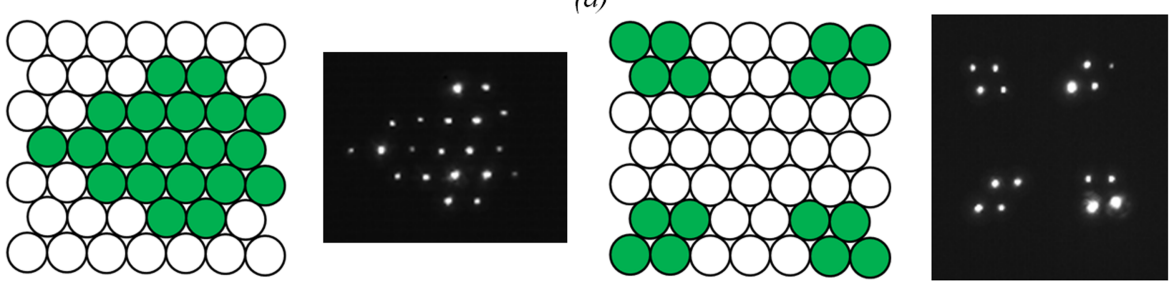

(b)

(c)
FIG. 12. 4-f optical configuration. (a) Photograph of the correlation system. Illuminated fibers (green) and image obtained at the NIR camera. (b) 20 fibers. (c) 16 fibers.

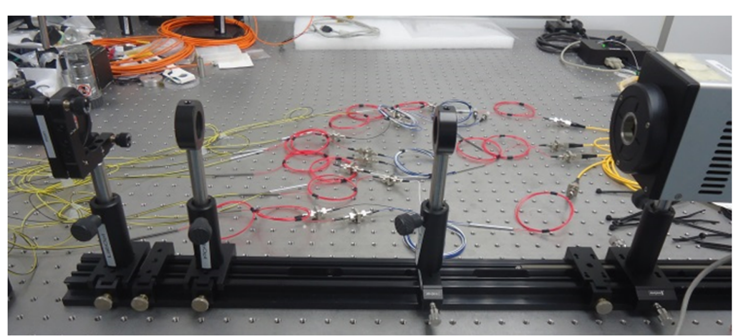

(a)

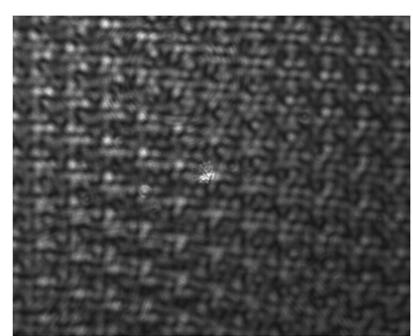

(b)
FIG. 13. 6-f optical configuration. (a) Photograph of the correlation system. (b) PSF (16 fibers). matching the optimal antenna array of the prototype. This is the reason to select a hexagonal package for both antennas and fibers.

In relation to the optical configuration of the lenses, we have used two different ones. A 4-f configuration was used to pass the optical beams through the lenses and reimage the fiber distribution illuminated in the bundle. This is useful for component alignment adjustments and other diagnostic purposes. As an example of this configuration, two different signal distributions were illuminated in the bundle. The first one consisted on illuminating a compact geometry of 20 fibers and the second using only 16 signals representing a possible distribution for the interferometer prototype with four receivers (Fig. 12).

With the implementation of the 4-f configuration, some differences in the intensity among signals were detected due to the existence of imperfections in some fibers within the bundle. Besides, it would be necessary to improve the fiber alignment in the bundle and the manufacture imperfections for future measurements.

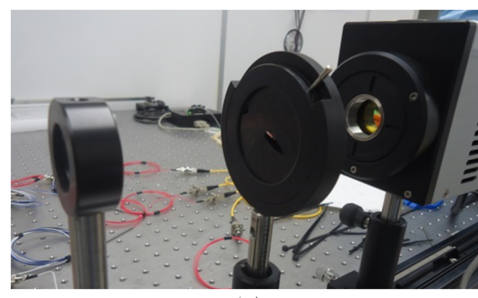

(a)

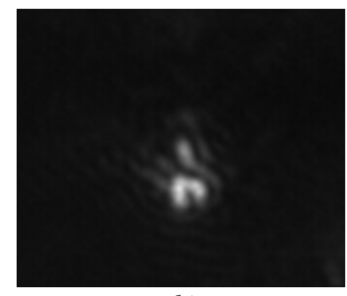

(b)
FIG. 14. Aberrations correction. (a) Inserted diaphragm. (b) PSF (16 fibers).
After obtaining the first images with the NIR camera, a 6-f configuration was implemented. This configuration permits to pass the optical beams through the lenses and synthesize the image of the sky region covered with the instrument. In the reported case, the point spread function (PSF) of the fiber array distribution can be observed. Fig. 13 shows the 6-f setup and the PSF obtained with the scenario detailed in Fig. 12(c).

PSF results from the previous figure show the existence of aberrations due to flaws in the optical elements that imply the degradation of the image obtained by the de NIR camera. In order to correct these aberrations, a diaphragm was introduced in the optical system to limit the light incident to the camera (Fig. 14(a)). The achieved PSF is shown in Fig. 14(b).

Future work related to the correlation stage performance analysis will consist of studying different PSF results obtained by changing the fiber distributions in the bundle once it is improved. Also, a more realistic setup using the up-converted signals from the modulators and an appropriated VBG optical filter will be implemented. Likewise, taking advance of the configuration possibilities of the NIR camera, it will be necessary to get the required configuration to achieve an optimum operation of the correlation stage. Finally, the utility of implementing some kind of electronic phase-control to implement an imager focusing method will be also analysed.

\section{CONCLUSION}

An up-conversion stage and preliminary signal correlation measurements for an electro-optic correlator prototype, with application to microwave large format interferometry, 
have been described. The proposed prototype will obtain the synthetized images of the Stokes parameters of an incoming wide-band linearly polarized signal by means of a real-time measurement in the NIR range.

Two different modulator operation point stabilization techniques in order to achieve good levels of optical carrier suppression during large periods of time have been reported. The stabilized modulator exhibited good performance obtaining $46 \mathrm{~dB}$ carrier suppression in comparison with the case in which the modulator was not DC biased. With this configuration, it was achieved a stable carrier level $23 \mathrm{~dB}$ lower than the signal of interest in the NIR spectrum.

On the other hand, a simplified correlation stage, based on the use of a two $100 \mathrm{~mm}$ focus lenses, has been presented. The measurements reported here provided information about the antenna array distribution in the fiber bundle and the PSF related to the selected fiber array configuration. Preliminary results of this correlation stage show that it would be necessary to correct some fiber bundle manufacturing errors, in order to reduce the measurement uncertainties before starting with the PSF analysis to achieve the optimal antenna/fiber array configuration.

\section{ACKNOWLEDGMENTS}

This work was supported by the Ministerio de Economía y Competitividad, Spain, under the Plan Nacional de I+D+i project with Reference No. ESP2015-70646-C2-1-R cofinanced with EU FEDER funds and the CONSOLIDERINGENIO 2010 programme under the Reference No. CSD2010-00064. The authors would like to thank Félix Gracia from Instituto Astrofísico de Canarias (IAC) for his assistance and help.

${ }^{1}$ R. A. Alpher, H. Bethe, and G. Gamow, Phys. Rev. 73, 803 (1948).

${ }^{2}$ A. A. Penzias and R. W. Wilson, Astrophys. J. 142, 419 (1965).

${ }^{3}$ I. Buder, "Q/U imaging experiment (QUIET): A ground-based probe of cosmic microwave background polarization," Proc. SPIE 7741, 77411D (2010).

${ }^{4}$ D. Barkats, R. Aikin, C. Bischoff, I. Buder, J. P. Kaufman, B. G. Keating, J. M. Kovac, M. Su, P. A. R. Ade, J. O. Battle, E. M. Bierman, J. J. Bock, H. C. Chiang, C. D. Dowell, L. Duband, J. Filippini, E. F. Hivon, W. L. Holzapfel, V. V. Hristov, W. C. Jones, C. L. Kuo, E. M. Leitch, P. V. Mason, T. Matsumura, H. T. Nguyen, N. Ponthieu, C. Pryke, S. Richter, G. Rocha,
C. Sheehy, S. S. Kernasovskiy, Y. D. Takahashi, J. E. Tolan, and K. W. Yoon, Astrophys. J. 783, 67 (2014).

${ }^{5}$ P. A. R. Ade, R. W. Aikin, D. Barkats, S. J. Benton, C. A. Bischoff, J. J. Bock, J. A. Brevik, I. Buder, E. Bullock, C. D. Dowell, L. Duband, J. P. Filippini, S. Fliescher, S. R. Golwala, M. Halpern, M. Hasselfield, S. R. Hildebrandt, G. C. Hilton, V. V. Hristov, K. D. Irwin, K. S. Karkare, J. P. Kaufman, B. G. Keating, S. A. Kernasovskiy, J. M. Kovac, C. L. Kuo, E. M. Leitch, M. Lueker, P. Mason, C. B. Netterfield, H. T. Nguyen, R. O'Brient, R. W. Ogburn IV, A. Orlando, C. Pryke, C. D. Reintsema, S. Richter, R. Schwarz, C. D. Sheehy, Z. K. Staniszewski, R. V. Sudiwala, G. P. Teply, J. E. Tolan, A. D. Turner, A. G. Vieregg, C. L. Wong, and K. W. Yoon, Phys. Rev. Lett. 112, 241101 (2014).

${ }^{6}$ J. A. Rubiño-Martín, R. Rebolo, M. Aguiar, R. Génova-Santos, F. GómezReñasco, J. M. Herreros, R. J. Hoyland, C. López-Caraballo, A. E. Pelaez Santos, V. Sanchez de La Rosa, A. Vega-Moreno, T. Viera-Curbelo, E. Martínez-Gonzalez, R. B. Barreiro, F. J. Casas, J. M. Diego, R. FernándezCobos, D. Herranz, M. López-Caniego, D. Ortiz, P. Vielva, E. Artal, B. Aja, J. Cagigas, J. L. Cano, L. De La Fuente, A. Mediavilla, J. V. Terán, E. Villa, L. Piccirillo, R. Battye, E. Blackhurst, M. Brown, R. D. Davies, R. J. Davis, C. Dickinson, S. Harper, B. Maffei, M. McCulloch, S. Melhuish, G. Pisano, R. A. Watson, M. Hobson, K. Grainge, A. Lasenby, R. Saunders, and P. Scott, Proc. SPIE 8444, 84442Y (2012).

${ }^{7}$ M. López-Caniego et al., "Instrumentation and methods for astrophysics," in Conference Proceedings Rencontres du Vietnam 2013: Cosmology in the Planck Era (ICISE, 2014), p. 1.

${ }^{8}$ N. W. Boggess, Highlights Astron. 9, 273 (1992); available at https://www. cambridge.org/core/journals/highlights-of-astronomy/article/the-cosmicbackground-explorer-cobe-mission-and-science-overview/CE964907562 ADAEAE8676F70E1999112.

${ }^{9}$ N. Jarosik, C. L. Bennett, J. Dunkley, B. Gold, M. R. Greason, M. Halpern, R. S. Hill, G. Hinshaw, A. Kogut, E. Komatsu, D. Larson, M. Limon, S. S. Meyer, M. R. Nolta, N. Odegard, L. Page, K. M. Smith, D. N. Spergel, G. S. Tucker, J. L. Weiland, E. Wollack, and E. L. Wright, Astrophys. J., Suppl. Ser. 192, 14 (2011).

${ }^{10}$ Planck Collaboration I, Astron. Astrophys. 536, 1 (2011).

${ }^{11}$ B. Aja, E. Artal, L. de la Fuente, J. P. Pascual, A. Mediavilla, N. Roddis, D. Kettle, W. F. Winder, L. Pradell, and P. de Paco, IEEE Trans. Microwave Theory Tech. 53, 2050 (2005).

${ }^{12}$ J. A. Grayson et al., Proc. SPIE 9914, 991401 (2016).

${ }^{13}$ B. Reichborn-Kjennerud et al., Proc. SPIE 7741, 774101 (2010).

${ }^{14}$ S. Aiola et al., Proc. SPIE 8446, 844601 (2012).

${ }^{15}$ C. Schuetz, R. Martin, T. Dillon, P. Yao, D. Mackrides, C. Harrity, A. Zablocki, K. Shreve, J. Bonnett, P. Curt, and D. Prather, Proc. SPIE 8715, 87150I (2013).

${ }^{16}$ J. Snoddy, Y. Li, F. Ravet, and X. Bao, Appl. Opt. 46, 1482 (2007).

${ }^{17}$ E. Villa, J. L. Cano, J. Cagigas, D. Ortiz, F. J. Casas, A. R. Pérez, B. Aja, J. V. Terán, L. de la Fuente, E. Artal, R. Hoyland, and A. Mediavilla, Rev. Sci. Instrum. 86, 024702 (2015).

${ }^{18}$ F. J. Casas, D. Ortiz, E. Villa, J. L. Cano, J. Cagigas, A. R. Pérez, B. Aja, J. V. Terán, L. de la Fuente, E. Artal, R. Hoyland, and R. Génova-Santos, Sensors 15, 19124 (2015)

${ }^{19}$ D.W. Prather, Photonic Materials and Devices for RF $(\mathrm{mmW})$ Sensing and Imaging (University of Delaware, 2012). 